\title{
Critical sets in latin squares and associated structures
}

\author{
RICHARD WINSTON BEAN
}

A critical set in a Latin square of order $n$ is a set of entries in an $n \times n$ array which can be embedded in precisely one Latin square of order $n$, with the property that if any entry of the critical set, is deleted, the remaining set can be embedded in more than one Latin square of order $n$.

The number of critical sets grows super-exponentially as the order of the Latin square increases. It is difficult to find patterns in Latin squares of small order (order 5 or less) which can be generalised in the process of creating new theorems. Thus, I have written many algorithms to find critical sets with various properties in Latin squares of order greater than 5 , and to deal with other related structures. Some algorithms used in the body of the thesis are presented followed by results which arise from the computational studies and observations of the patterns and subsequent results.

The cardinality of the largest critical set in any Latin square of order $n$ is denoted by $\operatorname{lcs}(n)$. In 1978 Curran and van Rees proved that $\operatorname{lcs}(n) \leqslant n^{2}-n$. In this thesis, it is shown that $\operatorname{lcs}(n) \leqslant n^{2}-3 n+3$.

I provide new bounds on the maximum number of intercalates in Latin squares of orders $2^{\alpha} m$ ( $m$ odd, $\alpha \geqslant 2$ ) and $2^{\alpha} m+1$ ( $m$ odd, $\alpha \geqslant 2$ and $\alpha \neq 3$ ), and a new lower bound on $\operatorname{lcs}(4 \mathrm{~m})$. I also discuss critical sets in intercalate-rich Latin squares of orders 11 and 14 .

A construction is given which verifies the existence of a critical set of size $\left(n^{2} / 4\right)+1$ when $n$ is even and $n \geqslant 6$. This construction is based on the discovery of a critical set of size 17 for a Latin square of order 8 .

The representation of Steiner trades of volume less than or equal to nine is examined. Computational results are used to identify those trades for which the associated partial Latin square can be decomposed into six disjoint Latin interchanges.

Finally, extensive computational routines are used to enumerate all critical sets in Latin squares of order at most six.

Papers based on chapters in this thesis have appeared in [4] and [5], are to appear in [3] and [2], and one has been submitted as [1].

Received 28th May, 2002

Thesis submitted to The University of Queensland, April 2001. Degree approved, November 2001. Supervisors: Dr Diane Donovan and Dr Elizabeth Billington.

Copyright Clearance Centre, Inc. Serial-fee code: 0004-9727/02 \$A2.00+0.00. 


\section{REFERENCES}

[1] P. Adams, R. Bean and A. Khodkar, 'A census of critical sets in the latin squares of order at most six', (submitted).

[2] P. Adams, R. Bean and A. Khodkar, 'Disjoint critical sets in latin squares.', Congr. Numer. (to appear).

[3] R. W. Bean and E. S. Mahmoodian, 'On the size of the largest critical set in a latin square', Discrete Math. (to appear).

[4] R. Bean and D. Donovan, 'Closing a gap in the spectrum of critical sets', Australas. J. Combin. 22 (2000), 199-210.

[5] R. Bean, D. Donovan, A. Khodkar and A. Penfold-Street., 'Steiner trades that give rise to completely decomposable latin interchanges', Int. J. Comput. Math. (to appear).

Department of Mathematics

The University of Queensland

Queensland 4072

Australia 